the student to learn the subject. He emphasized the value of an actual clinical test, for after thirty years' experience as an examiner, he knew how often candidates were well versed in book knowledge, but unable to diagnose the simplest ocular abnormality.

He said that the General Medical Council should be urged to institute the following reforms:

1. That each candidate for a licence to practise be compelled to attend an ophthalmic clinic for three months.

2. That the final examination shall include a clinical examination by ophthalmic surgeons.

\title{
A new Diploma in Ophthalmic Medicine and Surgery
}

A forward step has been taken by the London Conjoint Board in instituting a diploma in ophthalmic medicine and surgery (D.O.M.S., R.C.P. \& S. Eng.). Candidates may enter for Part I of the examination, which includes the anatomy and embryology of the visual apparatus, the physiology of vision, and elementary optics, at any time after a registrable qualification has been obtained in medicine, surgery, and midwifery. Part II, upon optical defects of the eye, ophthalmic medicine and surgery, and pathology, may be taken on the completion of one year of special study of ophthalmology, and the production of certain certificates. The institution of this diploma amply justifies the efforts of those who have advocated the adequate study of ophthalmology and furnishes the best answer to the attitude of the General Medical Council towards the matter. We congratulate the Royal Colleges upon their enlightened action.

\section{Artificial Daylight}

The problem of artificial daylight is one of considerable importance in many industries. One of the recent attempts at solving the problem consists in the use of carefully combined colour reflectors in contrast to the earlier methods in which transmission through tinted screens was employed. The apparatus is known as the Sheringham daylight and is the result of the combined work of a physicist, Mr. L. C. Martin, an artist, Mr. G. Sheringham, and an expert on camouflage problems, Major Klein. Mr. Martin read a paper on the light before the Illuminating Engineering Society. The paper and the subsequent interesting discussion are fully reported in the Illuminating Engineer for February, 1920. One very interesting point was the difficulty of defining daylight on account of its great variability. In an editorial in the same journal the editor writes: "In order that the whole subject may 